\title{
Immunohistochemical Characteristics of Renomedullary Interstitial Cell Tumor: A Study of 41 Tumors with Emphasis on Differential Diagnosis of Mesenchymal Neoplasms
}

\section{Authors:}

Zhichun Lu MD ${ }^{1}$ Department of Pathology \& Laboratory Medicine, Henry Ford Health System, Detroit, Michigan, USA

Khaleel Al-Obaidy MD ${ }^{2}$ Department of Pathology \& Laboratory Medicine, Indiana University School of Medicine, Indiana, USA

Liang Cheng MD ${ }^{2}$ Department of Pathology \& Laboratory Medicine, Indiana University School of Medicine, Indiana, USA

Kyle D Perry MD ${ }^{1}$ Department of Pathology \& Laboratory Medicine, Henry Ford Health System, Detroit, Michigan, USA

David J Grignon MD ${ }^{2}$ Department of Pathology \& Laboratory Medicine, Indiana University School of Medicine, Indiana, USA

Sean R Williamson MD ${ }^{1}$ Department of Pathology \& Laboratory Medicine, Henry Ford Health System, Detroit, Michigan, USA

Address correspondence and reprint requests to:

Sean R. Williamson, MD

Henry Ford Hospital, Department of Pathology - K6

2799 West Grand Blvd

Detroit, MI 48202

Email: swilli25@hfhs.org

Phone: +1(313)916-3986

Fax: $+1(313) 916-2385$

Conflict of Interest and Source of Funding: None

This is the author's manuscript of the article published in final edited form as:

Lu, Z., Al-Obaidy, K., Cheng, L., Perry, K. D., Grignon, D. J., \& Williamson, S. R. (2018). Immunohistochemical characteristics of Renomedullary interstitial cell tumor: a study of 41 tumors with emphasis on differential diagnosis of mesenchymal neoplasms. Human Pathology. https://doi.org/10.1016/j.humpath.2018.07.010 


\section{Abstract:}

Renomedullary interstitial cell tumors (RMICT) are almost always incidentally identified either at autopsy or resection of the kidney for other reasons. However, rare cases have been reported which are large, resulting in a clinical mass. The immunohistochemical phenotype of usual, incidental RMICT using modern soft tissue tumor markers in is largely unknown, however, providing little information to aid in classification of larger or atypical tumors. We retrieved 41 RMICTs from 36 patients, and studied pathologic characteristics including morphology, immunohistochemistry (S100, keratin AE1/AE3, smooth muscle actin, desmin, estrogen and progesterone receptors, calponin, CD34, CD35), and histochemical staining. Data collected included age, gender, tumor size, laterality, and indication for kidney examination. RMICTs $(n=41)$ were identified in 23 men and 13 women, with mean age 57 years (range 24-83), tumor sizes ranged from $<1$ to $13 \mathrm{~mm}$ (median $4 \mathrm{~mm}$ ). Kidneys were resected for 32 tumors, 1 chronic pyelonephritis, 1 trauma, and 2 autopsies. All $(41,100 \%)$ had entrapped renal tubules, 5 (12\%) of which included cystic or dilated tubules. Most $(35,85 \%)$ had collagenous fibers, all of which were negative for Congo red. RMICT demonstrates a largely negative immunohistochemical phenotype with weak to moderate labeling for smooth muscle actin and calponin that is substantially less than myofibroblastic lesions. Positive staining for estrogen and progesterone receptor is common (61\%), which could overlap with mixed epithelial and stromal tumor and other entities; however, staining is typically weak. CD34 is usually negative, with occasional weak labeling, in contrast to solitary fibrous tumor. 


\section{Keywords:}

Renomedullary interstitial cell tumor; kidney; renal mesenchymal tumors; medullary fibroma; solitary fibrous tumor; mixed epithelial and stromal tumor

Running head: Renomedullary interstitial cell tumor immunohistochemistry 


\section{Introduction:}

Renomedullary interstitial cell tumors are almost always incidentally identified either at autopsy or upon resection of the kidney for other reasons. Originally referred to as medullary fibroma, the terminology renomedullary interstitial cell tumor was proposed based on ultrastructural characteristics resembling renomedullary interstitial cells [1]. Given their characteristically small size (usually less than $5 \mathrm{~mm}$ ), renal medullary location, and light microscopic features, the differential diagnosis is typically quite limited. However, rare cases have been reported which are large or clinically present as a mass lesion [2-6]. Since the immunohistochemical profile of these lesions has not been thoroughly studied, especially using modern soft tissue tumor markers, a combination of immunohistochemical characteristics that would lend support to interpretation of such unusual or large lesions as renomedullary interstitial cell tumor is not known. As such, we sought to investigate the immunohistochemical and histochemical staining characteristics of renomedullary interstitial cell tumors to aid in these circumstances and shed light on their pathogenesis and relationship to soft tissue type neoplasms of the kidney.

\section{Materials and Methods}

\subsection{Study Design and Patient Selection}

After approval of the institutional review boards of the Henry Ford Health System and Indiana University School of Medicine, a total of 36 specimens from patients who underwent kidney examination for other reasons from 2001 to 2013 were retrieved from the archives of the Department of Pathology at Indiana University Health. Of the original 39 cases retrieved, 3 were no longer present on deeper sections and excluded. This left the study cohort of 41 tumors 
from 36 patients. Histology was reviewed by two authors (ZL and SRW), and a representative section was selected for a panel of immunohistochemical and histochemical staining, including S100, keratin AE1/AE3, smooth muscle actin, desmin, estrogen and progesterone receptors, calponin, CD34, CD35, and Congo red. Clinical data collected included age, gender, tumor size, laterality, and indication for kidney examination.

\subsection{Histological Evaluation and Immunohistochemical staining}

Formalin-fixed paraffin-embedded tissues were sectioned $(4 \mu \mathrm{m})$, and stained for immunohistochemistry, using antibodies against: S100 (polyclonal; Dako Corp), cytokeratin AE1/AE3 (AE1/AE3; Dako Corp), smooth muscle actin (1A4; Dako Corp), desmin (D33; Dako Corp), estrogen receptor (EP1; Dako Corp), progesterone receptor (PgR636; Dako Corp), calponin (EP798Y; Cell Marque), CD34 (QBEnd; Dako Corp), and CD35 (RLB25; Cell Marque) were utilized in a Dako automated instrument. Congo red stain was performed using the Artisan staining kit (Dako Corp). Positive and negative controls gave appropriate results for each procedure. Faint staining requiring high magnification to appreciate was interpreted as $1+$. Moderate staining at least partly appreciable at $10 \times$ magnification was interpreted as $2+$, and strong, uniform staining apparent from low magnification was interpreted as 3+.

\section{Results}

\subsection{Clinical Features}

Reason for examination of 36 kidneys included: 32 removed for tumors, 1 for chronic pyelonephritis, 1 for trauma, and 2 autopsies. Tumors $(n=41)$ were present in 36 patients (23 male and 13 female patients, $M: F=1.8: 1$ ). (Table 1 summarizes the clinicopathological data of the 41 renomedullary interstitial cell tumors). Patients' ages ranged from 24-83 years (mean 
age: 57 years), lesion size range from $<1$ to $13 \mathrm{~mm}$ (median: $4 \mathrm{~mm}$ ). Coexistent neoplasms ( $n=34$, with one patient having 2 neoplasms) included 19 clear cell renal cell carcinomas, 3 renal cell carcinomas unclassified, 2 angiomyolipomas, 2 adrenal cortical carcinomas, 1 chromophobe renal cell carcinoma, 1 urothelial carcinoma, 1 papillary renal cell carcinoma, 1 oncocytoma, 1 well differentiated liposarcoma, 1 mixed epithelial and stromal tumor, 1 adrenal gland malignant solitary fibrous tumor, and 1 metastatic pulmonary squamous cell carcinoma.

\subsection{Histologic Features}

Tumors were characteristically small (median: $4 \mathrm{~mm}$ ). All lesions contained some degree of entrapped normal renal tubules. In these, 22/41 (53\%) renomedullary interstitial cell tumors contained diffusely entrapped renal tubules, whereas 19/41 (47\%) renomedullary interstitial cell tumors contained peripherally entrapped tubules. Cystic dilated tubules were present in in $5 / 41$ (12\%). Eosinophilic collagen bundles were present in 35/41 (85\%). The tumor cells were small, stellate, and spindle-shaped with bland nuclei, embedded in a faintly basophilic stroma.

\subsection{Immunohistochemical and histochemical Features}

A selected immunohistochemical and histochemical staining panel was performed on 41 renomedullary interstitial cell tumors, including S100, keratin AE1/AE3, smooth muscle actin, desmin, estrogen receptor, progesterone receptor, calponin, CD34, CD35, and Congo red (Table 2). Smooth muscle actin, estrogen receptor, progesterone receptor, calponin and CD34 showed slightly varied immunoreactivity in renomedullary interstitial cell tumor cells. Many (29/41) showed positive immunohistochemical staining for smooth muscle actin (Figure 1), of which, 17 cases showed weak positivity $(1+), 11$ cases moderate intensity positivity $(2+)$, and 1 focal strong positivity (focal $3+$ ). The majority $(39 / 41,95 \%)$ of renomedullary interstitial cell tumors 
were positive for calponin (Figure 1). In such cases, 32/39 (82\%) showed moderate positivity $(2+), 2 / 39$ showed weak positivity (1+). However, their overall intensity was sometimes weaker than that of adjacent interstitial cells. One case in this series had an angiomyolipoma adjacent to the renomedullary interstitial cell tumor which showed strong immunohistochemical positivity for both smooth muscle actin and desmin, in contrast to the renomedullary interstitial cell tumors.

Estrogen and progesterone receptors both showed positive staining in $61 \%$ of renomedullary interstitial cell tumors (25/41) with weak $(1+)$ to moderate $(2+)$ intensity (Figure 2 ). The staining for estrogen and progesterone receptors was also observed in the adjacent renal interstitial cells with similar intensity (Figure 2). A minority of cases (10/41, 24\%) was positive for CD34, of which 8 lesions were weakly positive (1+) and 2 were focally positive with moderate intensity (2+). S100, keratin AE1/AE3, desmin, and CD35 were consistently negative. Congo red was negative for amyloid in all renomedullary interstitial cell tumors.

\section{Discussion}

Renomedullary interstitial cell tumor is typically a straightforward diagnosis, due to the morphology, medullary location, and small size. In the vast majority of cases, this is an incidental diagnosis of minimal to no significance, encountered at kidney examination for other reasons.[7] However, we have occasionally encountered lesions that are larger or cellular, raising a differential diagnosis of other entities, such as mixed epithelial and stromal tumor (when entrapped or cystic tubules are present), angiomyolipoma, or solitary fibrous tumor. Likewise, occasional case reports and small series have reported larger tumors thought to be renomedullary interstitial cell tumor that were clinically mass-forming, being an indication for 
surgery themselves [2-6]. These studies have reported immunohistochemical staining results in these larger tumors; however, since there is scant information on the immunohistochemical staining characteristics of usual, small renomedullary interstitial cell tumor, the expected immunohistochemical phenotype is not well established to serve as a comparison for larger or atypical cases. Therefore, in this study, we used a panel of immunohistochemical markers relevant to soft tissue tumor pathology to investigate the phenotype of renomedullary interstitial cell tumor. We found that renomedullary interstitial cell tumor demonstrates a largely negative immunohistochemical phenotype for markers including desmin, S100, and cytokeratin AE1/AE3. Although we found many tumors to exhibit positive immunohistochemical staining for smooth muscle actin and calponin, this was quite weak in comparison to smooth muscle tumors or angiomyolipoma. Although a prior study noted staining for CD35 in renomedullary interstitial cell tumors [8], this was not reproduced in our study, possibly reflecting technical differences, such as antibody clone or staining parameters.

Potential differential diagnoses for renomedullary interstitial cell tumor could include mixed epithelial and stromal tumor, for tumors that are larger with a cystic tubular component. In this series, cystic tubules were present in 5/41 (12\%) of renomedullary interstitial cell tumors. Although we found staining for estrogen and progesterone receptor to be positive in renomedullary interstitial cell tumor relatively commonly $(61 \%, 25 / 41)$, this was very limited in intensity, with almost all cases exiting not more than weak (1+) staining. As such, the quantitative amount of staining for these markers may be potentially different from mixed epithelial and stromal tumor. Of note, although staining for these hormone receptors is a prototypical feature of mixed epithelial and stromal tumor, it may not be entirely specific. One 
study by Tickoo et al found immunohistochemical positivity for estrogen and progesterone receptors in renal interstitial cells, which was thought to represent a metaplastic change in association with obstruction [9]. Other differential diagnostic considerations for larger renomedullary interstitial cell tumors that are composed of a mixture of spindle-shaped cells and collagen could include solitary fibrous tumor [10]. Although we found some positive staining for CD34 in $24 \%$ of renomedullary interstitial cell tumors (10/41), again the intensity was typically minor, contrasting to the usual pattern of solitary fibrous tumor. The morphology of renomedullary interstitial cell tumor is usually quite different from that of angiomyolipoma, in that the spindle-shaped cells typically have scant cytoplasm, in contrast to the eosinophilic, fibrillary, or clear cytoplasm of angiomyolipoma; however, this could nonetheless also be a consideration for larger or cellular lesions, including angiomyolipoma with entrapped cysts (AMLEC) or other unusual patterns of angiomyolipoma. The limited intensity of smooth muscle actin staining in renomedullary interstitial cell tumor is a contrast to angiomyolipoma. Indeed, one case in this series had an angiomyolipoma adjacent to the renomedullary interstitial cell tumor, in which smooth muscle actin and desmin staining were strong, contrasting to the renomedullary interstitial cell tumor. Other extremely rare renal mesenchymal neoplasms that could be potentially considered include leiomyoma and schwannoma, which differ in their immunohistochemical staining for smooth muscle actin and desmin and S100 staining, respectively.

In conclusion, renomedullary interstitial cell tumor demonstrates a largely negative immunohistochemical phenotype with weak to moderate labeling for smooth muscle actin and calponin that is substantially less than myofibroblastic or smooth muscle lesions. Positive 
staining for estrogen and progesterone receptor is common, which could overlap with mixed epithelial and stromal tumor and other entities; however, this staining is typically weak, a point of contrast to mixed epithelial and stromal tumor. CD34 staining is usually negative, with occasional weak labeling, which contrasts to solitary fibrous tumor.

\section{Acknowledgements}

No funding was received for this work.

\section{Figure Legends:}

1. This renomedullary interstitial cell tumor (A, 100x, hematoxylin and eosin) contains a large, dilated tubule, which might impart a resemblance to a small mixed epithelial and stromal tumor. Staining for smooth muscle actin (B, 100x, anti-smooth muscle actin immunohistochemistry and inset, 400x) is positive in part of the lesion at top but negative in other areas. Another case (C, 100x, hematoxylin and eosin and inset, 400x) is more cellular, with small entrapped tubules. This example exhibits weak staining for calponin ( $D, 100 x$, anti-calponin immunohistochemistry) that is substantially weaker than adjacent smooth muscle (left). The weak staining is better visualized at high magnification (inset 400x).

2. Another renomedullary interstitial cell tumor ( $A, 100 x$, hematoxylin and eosin) is adjacent to a vein at right. Staining for estrogen receptor $(B, 100 \times$, anti-estrogen receptor immunohistochemistry) is stronger in the adjacent nontumor interstitial cells (inset, 400x) and perivascular tissue than in the lesion (C, 400x, anti-estrogen receptor immunohistochemistry). A different case ( $D, 400 x$, anti-estrogen receptor immunohistochemistry) shows slightly stronger staining in the renomedullary interstitial cell tumor. 


\section{References:}

1. Lerman RJ, Pitcock JA, Stephenson P, Muirhead EE. Renomedullary interstitial cell tumor (formerly fibroma of renal medulla). Hum Pathol 1972; 3, 559-68.

2. Ohkawa M, Nonomura A, Shoda R. Renal medullary fibroma: a case report and review. Int Urol Nephrol 1993; 25, 45-9.

3. Mai KT. Giant renomedullary interstitial cell tumor. J Urol 1994; 151, 986-8.

4. Dall'Era M, Das S. Benign medullary fibroma of the kidney. J Urol 2000; 164, 2018.

5. Faris G, Nashashibi M, Friedman B, Stein A, Sova Y, Mecz Y. Urosepsis as a presenting symptom of renomedullary interstitial cell tumor causing renal obstruction. Isr Med Assoc J 2009; 11, 509-10.

6. Bazzi WM, Huang H, Al-Ahmadie H, Russo P. Clinicopathologic features of renomedullary interstitial cell tumor presenting as the main solid renal mass. Urology 2014; 83, 1104-6.

7. Calio A, Warfel KA, Eble JN. Renomedullary Interstitial Cell Tumors: Pathologic Features and Clinical Correlations. Am J Surg Pathol 2016; 40, 1693-701.

8. Kuroda N, Toi M, Miyazaki E, Hayashi Y, Nakayama H, Hiroi M, et al. Participation of alphasmooth muscle actin-positive cells in renomedullary interstitial cell tumors. Oncol Rep 2002; 9, 745-50.

9. Tickoo SK, Gopalan A, Tu JJ, Harik LR, Al-Ahmadie HA, Fine SW, et al. Estrogen and progesteronereceptor-positive stroma as a non-tumorous proliferation in kidneys: a possible metaplastic response to obstruction. Mod Pathol 2008; 21, 60-5.

10. Kuroda N, Ohe C, Sakaida N, Uemura Y, Inoue K, Nagashima Y, et al. Solitary fibrous tumor of the kidney with focus on clinical and pathobiological aspects. Int J Clin Exp Pathol 2014; 7, 2737-42. 
Table 1 Summary of Clinicopathological Characteristics of 41 Renomedullary Interstitial Cell

Tumors

\begin{tabular}{|l|l|}
\hline Clinicopathologic Parameters & Value \\
\hline Age & $24-83$ years (mean age: 57 years) \\
\hline Gender & 23 \\
$\quad$ Female & 13 \\
M:F ratio & $1.8: 1$ \\
\hline Size $\quad<1$ to 13 mm (median: 4 mm) \\
\hline Entrapped tubules & \\
Diffusely & $22(53 \%)$ \\
Peripherally & $19(47 \%)$ \\
Cystic tubules & $5(12 \%)$ \\
\hline Collagen bundles & $35(85 \%)$ \\
\hline Amyloid & 0 \\
\hline
\end{tabular}


Table 2: Summary of Immunohistochemical and Histochemical Features of 41 Renomedullary Interstitial Cell Tumors

\begin{tabular}{|l|l|l|l|l|l|}
\hline Stain & Negative - & Weak + & Moderate ++ & Strong +++ & Positivity (\%) \\
\hline S100 & 41 & 0 & 0 & 0 & 0 \\
\hline AE1/AE3 & 41 & 0 & 0 & 0 & 0 \\
\hline $\begin{array}{l}\text { Smooth muscle } \\
\text { actin }\end{array}$ & 12 & 17 & 11 & 1 & 71 \\
\hline Desmin & 41 & 0 & 0 & 0 & 0 \\
\hline ER & 16 & 22 & 3 & 0 & 61 \\
\hline PR & 16 & 23 & 2 & 0 & 61 \\
\hline Calponin & 2 & 6 & 33 & 0 & 95 \\
\hline CD34 & 31 & 8 & 2 & 0 & 24 \\
\hline CD35 & 41 & 0 & 0 & 0 & 0 \\
\hline Congo red & 41 & 0 & 0 & 0 & 0 \\
\hline
\end{tabular}




\section{Highlights}

- Renomedullary interstitial cell tumor is usually an incidental finding in kidney specimens

- Rare cases can be large, causing a clinical mass and raising differential diagnostic challenges

- The immunohistochemical profile of these tumors is largely negative

- Weak staining can be observed for muscle markers and hormone receptors (estrogen and progesterone) 

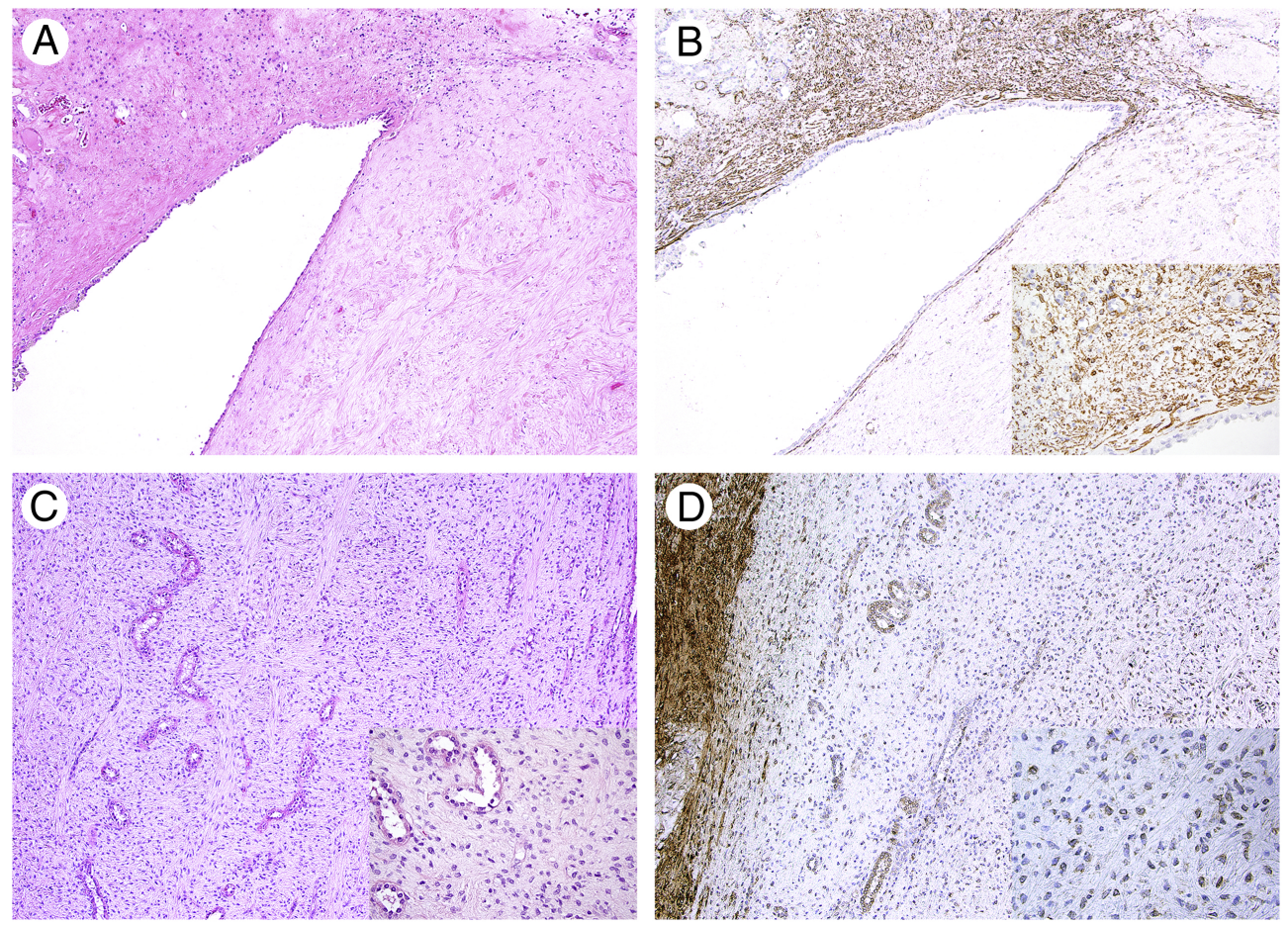

Figure 1 

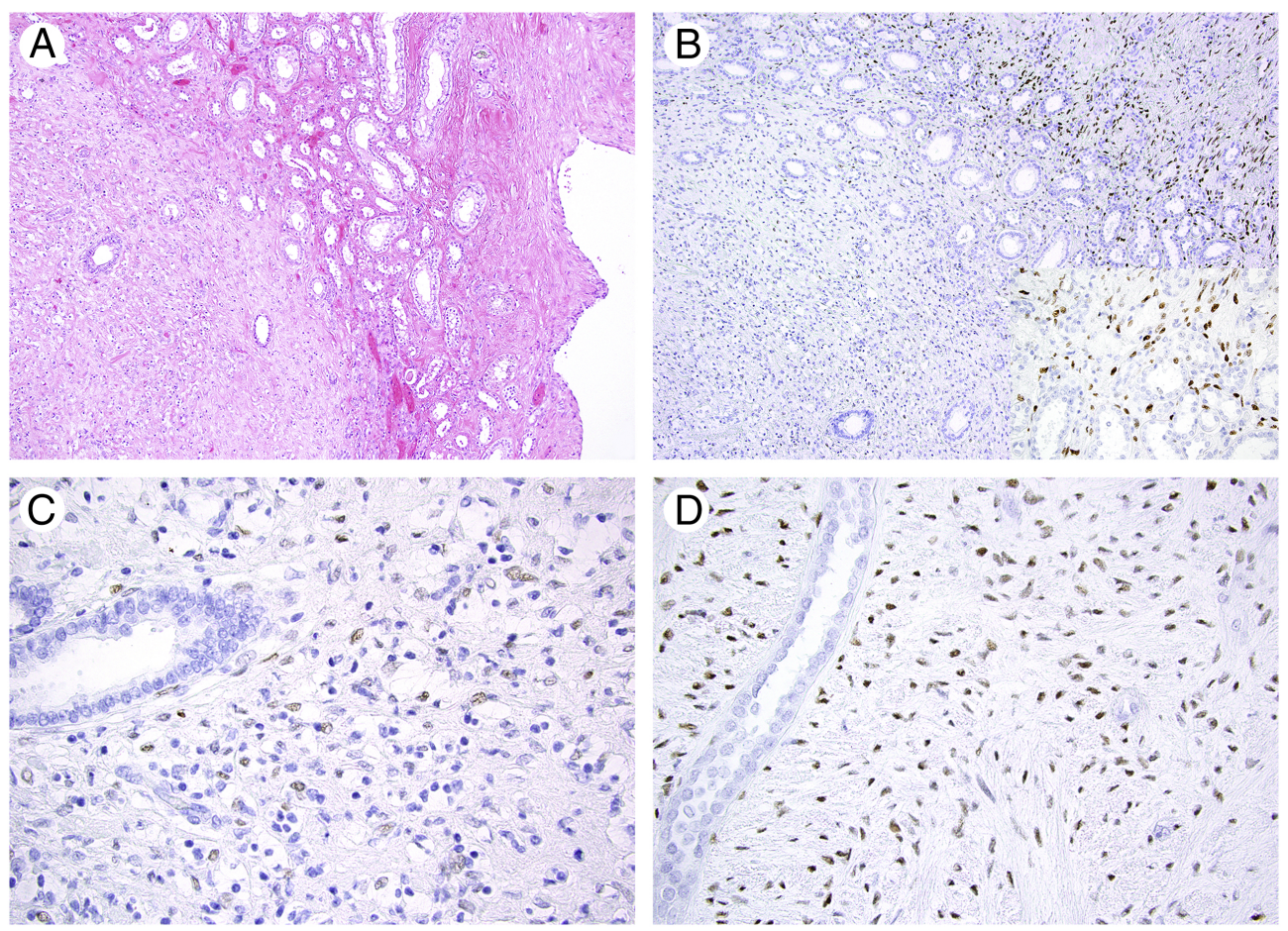

Figure 2 